\title{
Detection and prevalence of pathogenic Yersinia enterocolitica in refrigerated and frozen dairy products by duplex PCR and dot hybridization targeting the virF and ail genes
}

\author{
Y. W. Ye, ${ }^{* 1}$ N. Ling, ${ }^{*}$ Y. J. Han, ${ }^{*}$ and Q. P. Wu† ${ }^{1}$ \\ ${ }^{*}$ School of Biotechnology and Food Engineering, Hefei University of Technology, Hefei, 230009, China \\ †State Key Laboratory of Applied Microbiology, South China (the Ministry-Province Joint Development), \\ Provincial Key Laboratory of Microbiology Culture Collection and Application, Guangdong Institute of Microbiology, Guangzhou, 510070, China
}

\section{ABSTRACT}

Pathogenic Yersinia enterocolitica is involved in yersiniosis through expression of chromosome-borne or plasmid-borne virulence factors. Yersinia enterocolitica is a cold-tolerant pathogen frequently isolated from refrigerated or frozen foods. However, little attention has been focused on the prevalence of pathogenic $Y$. enterocolitica in refrigerated or frozen dairy samples in China. In this study, we developed a new duplex PCR targeting the plasmid-borne virF gene and chromosome-borne ail gene for detection of pathogenic $Y$. enterocolitica isolates. We established a detection limit for the duplex PCR of $6.5 \times 10^{2} \mathrm{cfu} / \mathrm{mL}$ in artificially contaminated dairy samples. In addition, the duplex PCR could detect directly 4.5 to $5.7 \mathrm{cfu}$ of $Y$. enterocolitica in $5 \mathrm{~mL}$ of brain heart infusion broth after 6 $\mathrm{h}$ of enrichment at $28^{\circ} \mathrm{C}$. A newly developed dot hybridization assay further confirmed specificity of the duplex PCR for detection of virulent $Y$. enterocolitica. Furthermore, $13 Y$. enterocolitica and 5 pathogenic strains, from 88 commercial frozen or refrigerated dairy products, were detected successfully by the China National Standard method (GB/T4789.8-2008) and the duplex PCR, respectively. Finally, biotypes and serotypes of pathogenic $Y$. enterocolitica strains were further characterized. The duplex PCR developed here is reliable for large-scale screening, routine monitoring, and risk assessment of pathogenic $Y$. enterocolitica in refrigerated or frozen dairy products.

Key words: pathogenic Yersinia enterocolitica, refrigerated or frozen dairy products, duplex PCR, dot hybridization

Received May 19, 2014.

Accepted August 8, 2014.

${ }^{1}$ Corresponding authors: yeyingwang04@126.com and wuqp203@163.

\section{INTRODUCTION}

Yersinia enterocolitica is a common foodborne pathogen causing acute gastroenteritis, enterocolitis, and mesenteric lymphadenitis as well as extraintestinal disorders in humans (Yucel and Ulusoy, 2006; Hanifian and Khani, 2012); it is ubiquitous in animals, foods, and environments (Fredriksson-Ahomaa and Korkeala, 2003). In Europe, yersiniosis is the third most important foodborne enteric infection after campylobacteriosis and salmonellosis (ESFA-ECDC, 2012; Tan et al., 2014). The first human yersiniosis was reported in Malaysia, where $Y$. enterocolitica $\mathrm{O}: 3$ was isolated from a 34-yr-old female (Jegathesan et al., 1984). Six biotypes of $Y$. enterocolitica (biotypes 1A, 1B, 2, 3, 4, 5) are known, and only biotype 1A strains are generally considered avirulent (Grant et al., 1998). The biovar/ serovar combination most often involved in human disease in Europe is 4/O:3, followed by 2/O:5, 27, 3/O:5, 27 (Gürtler et al., 2005). Nonpathogenic strains are frequently isolated from food samples, and it is therefore essential to distinguish truly pathogenic strains from apathogenic strains. The identification of pathogenic $Y$. enterocolitica is traditionally based on phenotypic tests such as calcium-dependent growth at $37^{\circ} \mathrm{C}$, Congo red binding (Prpic et al., 1983), pyrazinamidase testing (Kandolo and Wauters, 1985), autoagglutination testing, and serum-resistance testing (Bhaduri and Cottrell, 1997). However, serological and biochemical classifications are time consuming and have low specificity for pathogenic isolates of $Y$. enterocolitica (Thoerner et al., 2003).

The pathogenicity of $Y$. enterocolitica depends on expression of plasmid and chromosomal determinants (Kuhnert et al., 1997). The $Y$. enterocolitica attachment invasion locus gene (ail) is reported to be present in only pathogenic strains (Miller and Falkow, 1988; Miller et al., 1989). Plasmid-borne virulence factors include the virF gene, which encodes transcriptional activators of $Y$. enterocolitica outer membrane protein (yop) regulation and yadA gene involved in autoagglutination, serum resistance, and adhesion (Skurnik 
and Wolf-Watz, 1989). Therefore, rapid and accurate detection of pathogenic $Y$. enterocolitica should rely on virulent factors not only from the chromosome, but also from the plasmid.

Yersinia enterocolitica is able to survive and proliferate in frozen or refrigerated conditions and is widely distributed in milk samples (Schiemann, 1987; Yucel and Ulusoy, 2006; Hanifian and Khani, 2012). Frozen or refrigerated dairy products are popular with consumers. In China, 296 strains of $Y$. enterocolitica were collected from humans with diarrhea, pigs, rodents, sheep, and dogs between 1984 and 2011 for analysis of variation and changes in serotypes, biotypes, and genetic typing (Guo et al., 2012). In another paper, Huang et al. (2010) reported possible use of ail and foxA polymorphisms for detection of $Y$. enterocolitica strains isolated from humans with diarrhea, animals, and environments. However, information about prevalence of pathogenic $Y$. enterocolitica in frozen or refrigerated dairy products is not available.

The aim of this study was to develop a duplex PCR targeting the virF and ail genes for determination of prevalence of pathogenic $Y$. enterocolitica in refrigerated or frozen dairy products. In addition, we developed a dot hybridization assay based on virF or ail genes to further determine the specificity of the duplex PCR. Thereafter, the detection limits of the duplex PCR and dot hybridization were determined. Finally, virulent and avirulent $Y$. enterocolitica isolates were tested in commercial frozen or refrigerated dairy samples $(\mathrm{n}=$ 88 ) by the duplex PCR and the China National Standard (2008) method.

\section{MATERIALS AND METHODS}

\section{Strains Used in this Study}

All strains used in this study were from Guangdong Provincial Key Laboratory of Microbiology Culture Collection and Application (Guangzhou, China). Strains included $72 Y$. enterocolitica, including 2 pathogenic strains (CMCC 52204 and CY39), 22 Escherichia coli, 16 Salmonella, 1 Enterobacter cloacae, 16 Listeria monocytogenes, 9 Staphylococcus aureus, 10 Aeromonas hydrophila, and 6 Aeromonas veronii. Yersinia enterocolitica CY39 and the type strain CMCC 52204 were used as positive controls for detection of ail $^{+} / \mathrm{virF}^{+}$and $\mathrm{ail}^{+}$, respectively. The non-Yersinia enterocolitica strains were used as negative controls.

\section{Extraction of Genomic and Plasmid DNA of $Y$. enterocolitica and Other Strains}

All species and strains listed above were inoculated into brain heart infusion broth (BHI, Huankai, Guang- zhou, China) at $28^{\circ} \mathrm{C}$ for $36 \mathrm{~h}$ (for $Y$. enterocolitica) or $37^{\circ} \mathrm{C}$ for $24 \mathrm{~h}$ (for other strains), respectively. One milliliter of enrichment culture (about $6.5 \times 10^{9} \mathrm{cfu} /$ $\mathrm{mL}$ for $Y$. enterocolitica, and 3.5 to $7.8 \times 10^{9} \mathrm{cfu} / \mathrm{mL}$ for other species) was used for extraction of genomic and plasmid DNA using the nucleic acid purification kit (DSBIO, Guangzhou, China) and high plasmid extraction kit (DSBIO), respectively, according to the manufacturer's instructions. The DNA templates were dissolved in Tris-EDTA buffer $(30 \mu \mathrm{L})$ and stored at $-30^{\circ} \mathrm{C}$ until further use.

\section{Detection of Pathogenic Y. enterocolitica by Duplex PCR}

The primers were described previously by Thoerner et al (2003). For the duplex PCR, 2 pairs of primers were used: (forward): 5'-taatgtgtacgctgcgag-3', (reverse): $5^{\prime}$-gacgtcttacttgcactg-3' for ail amplification; and (forward): $5^{\prime}$-ggcagaacagcagtcagacata- $3^{\prime}$, (reverse): $5^{\prime}$-ggtgagcatagagaatacgtcg-3' for virF amplification. The PCR mixture $(25 \mu \mathrm{L})$ consisted of $1.0 \mu \mathrm{L}$ of primers $(10 \mu M)$ for each, $200 \mu M$ deoxynucleotide triphosphates, $2 \mathrm{U}$ of Taq DNA polymerase, $2.5 \mu \mathrm{L}$ of $10 \times$ buffer, $3 \mu \mathrm{L}$ of DNA template, and double-distilled water. The duplex PCR was performed as follows: denatured for $2.0 \mathrm{~min}$ at $95^{\circ} \mathrm{C}$, followed by 35 cycles of $1.0 \mathrm{~min}$ at $94^{\circ} \mathrm{C}, 45 \mathrm{~s}$ at $58^{\circ} \mathrm{C}$, and $1.0 \mathrm{~min}$ at $72^{\circ} \mathrm{C}$; and final extension at $72^{\circ} \mathrm{C}$ for $7.0 \mathrm{~min}$. The amplicons were detected by $1.5 \%$ agarose gel electrophoresis with Goldview staining (0.008\%, vol/vol, Beijing Solarbio Science \& Technology Co. Ltd., Beijing, China), and were photographed under a UV transilluminator.

\section{Labeling and Detection of Pathogenic Y. enterocolitica by Dot Hybridization}

The $\operatorname{virF}(561 \mathrm{bp})$ and ail (351 bp) sequences from the positive strains were labeled using the DIG High Prime DNA Labeling and Detection Starter Kit I (Roche, Basel, Switzerland) according to the manufacturer's instructions. The detection of dot hybridization was performed using Detection Starter Kit I (Roche) according to the manufacturer's instructions. In brief, $1.0 \mu \mathrm{L}$ of tested DNA template was spotted onto the nylon membrane, the membrane was placed onto 3-MM paper (Whatman PLC, Little Chalfont, UK) soaked with $2 \times$ saline sodium citrate, and the template DNA on wet membrane was fixed by crosslinking with UV light. After crosslinking, the membrane was rinsed briefly in double-distilled water and was allow to air dry. Thereafter, the membrane was transferred into a plastic container with DIG Easy Hyb $\left(10 \mathrm{~mL} / 100-\mathrm{cm}^{2}\right.$ filter; Roche) for prehybridization with gentle agitation 
for $30 \mathrm{~min}$. Prehybridization solution was poured off and mixture of denatured labeled probe and preheated DIG Easy Hyb $\left(3.5 \mathrm{~mL} / 100-\mathrm{cm}^{2}\right.$ filter $)$ was transferred onto the membrane for incubation for $4 \mathrm{~h}$. After hybridization and stringency washes, the membrane was briefly washed (5 min) in washing buffer, and then incubated in $10 \mathrm{~mL}$ of blocking solution for $30 \mathrm{~min}$ and $10 \mathrm{~mL}$ of antibody solution for $30 \mathrm{~min}$, washed with 10 $\mathrm{mL}$ of washing buffer twice for $15 \mathrm{~min}$ each time, and then equilibrated in $10 \mathrm{~mL}$ of detection buffer for 2 to $5 \mathrm{~min}$. Finally, the membrane was incubated in $2 \mathrm{~mL}$ of freshly prepared color nitro-blue tetrazolium chloride/ 5-bromo-4-chloro-3'-indolyphosphate $p$-toluidine salt (NBT/BCIP) substrate solution in a hybrid tube in the dark. When desired spot or band intensities were achieved, the membrane was washed for 5 min with 50 $\mathrm{mL}$ of Tris-EDTA buffer to stop the reaction, and then the membrane was documented by photography.

\section{Detection Limit for Artificially Contaminated Dairy Samples}

Refrigerated yogurt (1.0 g) negative for $Y$. enterocolitica by the China National Standard (2008) method was mixed with $8 \mathrm{~mL}$ of sterile distilled water in a tube. Serial 10-fold dilutions from $6.5 \times 10^{9}$ to $6.5 \times$ $10^{0} \mathrm{cfu} / \mathrm{mL}$ were made, and $1.0 \mathrm{~mL}$ of each dilution was added to $9.0 \mathrm{~mL}$ of reconstituted yogurt. One milliliter of artificially contaminated yogurt was subject to genomic and plasmid DNA extraction using the same kits as used extraction of DNA from bacterial species. Genomic and plasmid DNA were stored at $-30^{\circ} \mathrm{C}$ pending analysis. The PCR amplification and dot hybridization protocol were performed as described above.

\section{Detection Limit of PCR Combined with Nonselective Enrichment}

Brain heart infusion broth $(5 \mathrm{~mL})$ was inoculated with 4.5 to $5.7 \mathrm{cfu}$ of $Y$. enterocolitica CY39 and incubated at $28^{\circ} \mathrm{C}$ with shaking (180 rpm) for $2,4,6,8$, $10,12,14,16$, and $18 \mathrm{~h}$. The extraction of plasmid and genomic DNA from the different time-enrichment cultures $(1.0 \mathrm{~mL})$ and PCR amplification were performed as described above.

\section{Detection of Y. enterocolitica in Commercial Frozen or Refrigerated Dairy}

Eighty-eight frozen or refrigerated dairy samples including ice cream $(\mathrm{n}=20)$, refrigerated yogurt $(\mathrm{n}=$ 14), milk popsicles $(\mathrm{n}=18)$, refrigerated raw milk ( $\mathrm{n}$ $=14)$, and frozen cheese $(\mathrm{n}=22)$ were collected from supermarkets or retail shops. The samples $(25 \mathrm{~g})$ were incubated into $225 \mathrm{~mL}$ of modified phosphate saline buffer and incubated at $28^{\circ} \mathrm{C}$ for $48 \mathrm{~h}$. Then, $1 \mathrm{~mL}$ of culture was subjected to extraction of template DNA (genomic DNA and plasmid DNA). Yersinia enterocolitica and pathogenic $Y$. enterocolitica were isolated and detected by the China National Standard (2008) method and the duplex PCR method, respectively.

\section{Phenotyping of Pathogenic Y. enterocolitica Strains}

Yersinia enterocolitica phenotypes were grouped by biotyping (lipase, esculin, salicin, indole, xylose, and trehalose tests) and serotyped, using commercial serum, as O:3, O:5, O:8, O:9, and O:1, 2 (Denka Seiken, Tokyo, Japan) according to the manufacturer's instructions.

\section{RESULTS AND DISCUSSION}

Yersinia enterocolitica is a common foodborne pathogen causing human cases of yersiniosis through consumption of contaminated food (Tan et al., 2014). Consequently, detection of pathogenic $Y$. enterocolitica in frozen dairy samples is important in assuring microbial safety.

In this study, a duplex PCR targeting plasmid-borne vir $F$ and chromosome-borne ail genes was developed to simultaneously detect virulent $Y$. enterocolitica strains in frozen or refrigerated dairy samples. The expected fragments ( $561 \mathrm{bp}$ for $\operatorname{virF}, 351 \mathrm{bp}$ for ail) were successfully amplified from 12 out of $72 Y$. enterocolitica isolates, but not in any non- $Y$. enterocolitica strains by our new duplex PCR. In Figure 1, among 12 pathogenic $Y$. enterocolitica, 6 pathogenic $Y$. enterocolitica strains were positive for both ail and virF genes, and 6 strains were positive for the ail gene only. As seen in Figure 2, detection by dot hybridization was consistent with results detected by the duplex PCR, which further confirmed the specificity of the duplex PCR method for detection of virulent $Y$. enterocolitica. Using the dot hy-

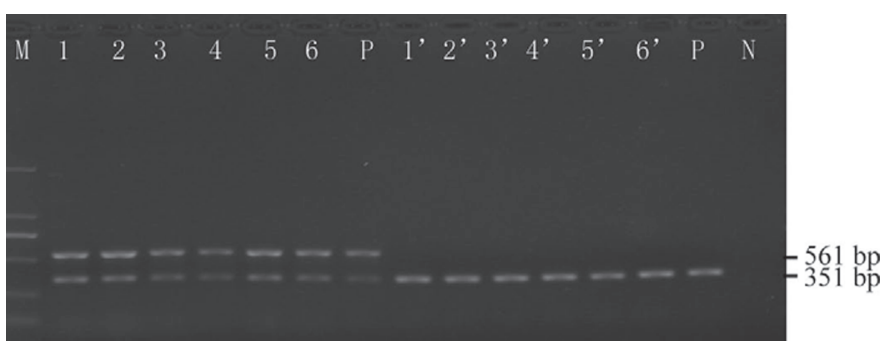

Figure 1. Detection of pathogenic Yersinia enterocolitica strains using duplex PCR. $\mathrm{M}=$ marker; lanes 1 to $6=$ pathogenic $Y$. enterocolitica strains containing both ail and vir $F$ genes; lanes $1^{\prime}$ to $6^{\prime}$ $=$ pathogenic $Y$. enterocolitica strains containing ail gene only; $\mathrm{P}=$ positive control, $\mathrm{N}=$ negative control. 

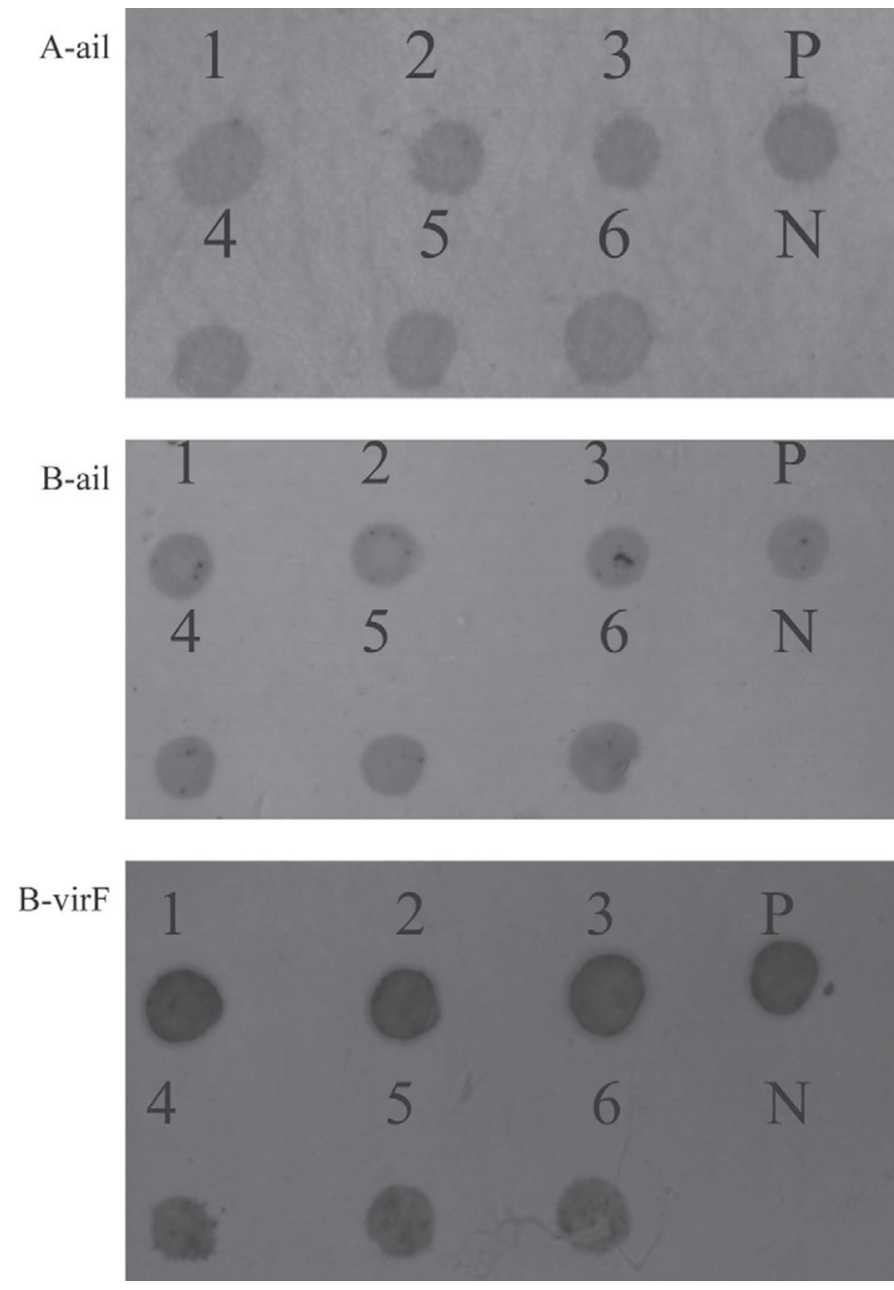

Figure 2. Detection of pathogenic Yersinia enterocolitica strains by dot hybridization assay on ail and virF genes. A-ail $=6$ pathogenic $Y$. enterocolitica strains containing ail gene only; B-ail and B-virF = 6 pathogenic $Y$. enterocolitica strains containing both ail and virF virulence genes; $\mathrm{P}=$ positive control, $\mathrm{N}=$ negative control. Dots 1 to 6 correspond to the 6 pathogenic $Y$. enterocolitica strains in each set. bridization assay, we achieved a detection limit of $6.5 \times$ $10^{3} \mathrm{cfu} / \mathrm{mL}$ (Figure 3A). A detection limit of $1,000 \mathrm{cfu}$ by microarray hybridization targeting virulence genes in pasteurized whole milk was described by Myers et al. (2006). As seen in Figure 3B, our duplex PCR could directly detect $6.5 \times 10^{2} \mathrm{cfu} / \mathrm{mL}$ of $Y$. enterocolitica in artificially contaminated dairy samples. A detection level of $4.0 \times 10^{2}$ cfu of $Y$. enterocolitica per $\mathrm{mL}$ of enrichment medium was obtained by a duplex PCR targeting yadA and $16 \mathrm{~S}$ rRNA genes in combination with the density gradient centrifugation method (Lantz et al., 1998). Nilsson et al. (1998) reported that as few as $10^{2}$ cfu could be successfully detected in ground pork in the presence of $10^{5}$ to $10^{6} \mathrm{cfu}$ of other species by a single PCR targeting ail or virF. However, in that study, only 16 of 25 pathogenic $Y$. enterocolitica strains simultaneously yielded positive results for both genes. To determine the influence of enrichment on the detection limit (Figure 4), the expected amplicons were amplified by the duplex PCR from the artificially dairy sample inoculated with 4.5 to $5.7 \mathrm{cfu}$ of $Y$. enterocolitica $/ 5 \mathrm{~mL}$ of brain heart infusion broth after $6 \mathrm{~h}$ of nonselective enrichment at $28^{\circ} \mathrm{C}$. In an earlier study using selective enrichment at $25^{\circ} \mathrm{C}$, a positive PCR result was obtained from selective enrichment medium with a background flora inoculated with approximately 1.0 $\times 10^{1} \mathrm{cfu} / \mathrm{mL} Y$. enterocolitica after 6 to $8 \mathrm{~h}$ at $25^{\circ} \mathrm{C}$ (Lantz et al., 1998). The current study also indicated that the duplex PCR was specific and sensitive for detection of pathogenic $Y$. enterocolitica when combined with an enrichment step.

Surveillance of $Y$. enterocolitica is important for risk analysis and assessment of frozen or refrigerated dairy products. In Pennsylvania, Y. enterocolitica was detected in $248(1.2 \%)$ milk samples (Jayarao et al., 2006). In northwestern Iran, $7.6 \%$ of bulk raw milks $(\mathrm{n}=354)$ and $10.5 \%$ of retail cheeses $(n=200)$ was found to be positive for ail by PCR (Hanifian and Khani, 2012). In Turkey, a survey indicated that $Y$. enterocolitica was

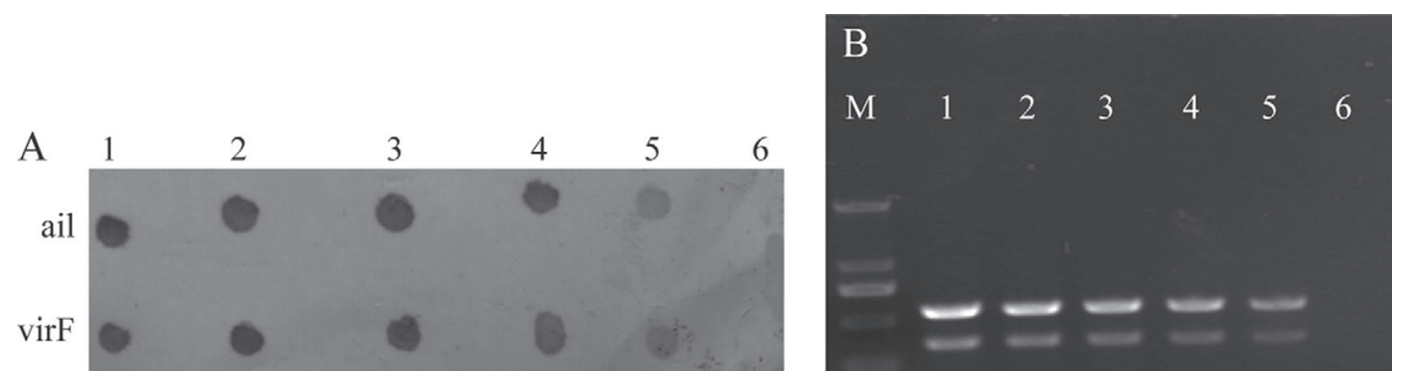

Figure 3. Detection limit of Yersinia enterocolitica strains in artificially contaminated dairy product by dot hybridization (A) and duplex PCR (B) for ail and virF genes. (A) dots 1 to $6=6.5 \times 10^{6}, 6.5 \times 10^{5}, 6.5 \times 10^{4}, 6.5 \times 10^{3}, 6.5 \times 10^{2}$, and $6.5 \times 10^{1}$ cfu/mL, respectively; (B) $\mathrm{M}=$ marker; lanes 1 to $6=6.5 \times 10^{7}, 6.5 \times 10^{6}, 6.5 \times 10^{5}, 6.5 \times 10^{4}, 6.5 \times 10^{3}$, and $6.5 \times 10^{2} \mathrm{cfu} / \mathrm{mL}$, respectively. 
isolated from $47.3 \%$ of raw milk samples and $35.7 \%$ of cheese samples (Yucel and Ulusoy, 2006). In the current study (Table 1), out of 88 dairy samples, pathogenic $Y$. enterocolitica was detected in $5(5.7 \%)$ samples, of which milk popsicles had the highest prevalence $(2 / 18$; $11.1 \%)$, followed by refrigerated raw milk $(1 / 14 ; 7.1 \%)$, ice cream $(1 / 20 ; 5 \%)$, frozen cheese $(1 / 22 ; 4.5 \%)$, and refrigerated yogurt $(0 / 14 ; 0.0 \%)$, compared with 16.7 , $15,14.3,14.3$, and $13.6 \%$ of milk popsicles, ice cream, refrigerated yogurt, refrigerated raw milk, and frozen cheese samples shown to be positive for $Y$. enterocolitica by the China National Standard (2008) method. In Table 2, phenotypic tests indicate that 17 strains were grouped into biotype 1B (7 isolates), biotype 2 (8 isolates), and biotype 3 (2 isolates). Miller et al. (1989) reported that $Y$. enterocolitica from biotypes 1B, $2,3,4$, and 5 were pathogenic in humans, which was consistent with our results. The following $\mathrm{O}$ serotypes were detected among these pathogenic $Y$. enterocolitica strains: O:8 (7 isolates), O:5 (4 isolates), O:3 (3 isolates), and $\mathrm{O}: 9$ (2 isolates), but 1 isolate was negative for the O serotype (O:3, O:5, O:8, O:9, and O:1, 2). Serotype groups associated with human infections (O:3, $\mathrm{O}: 5, \mathrm{O}: 8$, and $\mathrm{O}: 9)$ were previously described by Bottone (1999), but a clear correlation between biotype and $\mathrm{O}$ serotype was not observed in this study.

The survey in commercial dairy samples indicated that contamination of $Y$. enterocolitica is relatively severe and should be emphasized. In addition, only 1 of 5 pathogenic $Y$. enterocolitica strains bore both the ail and virF genes. Previous studies have reported that all $Y$. enterocolitica strains harboring the ail gene always carry the yst gene (Miller et al., 1989; Harnett et al., 1996; Myers et al., 2006). In present study, we found that the plasmid-borne virF did not occur alone in pathogenic $Y$. enterocolitica and was always accompanied by the ail gene. The developed duplex PCR was able to differentiate pathogenic $Y$. enterocolitica from apathogenic strains regardless of biotype or serotype. The serotypes O:3, O:5, O:8, and O:9 and biotypes $1 \mathrm{~B}, 2,3,4$, and 5 were considered pathogenic (Wan-

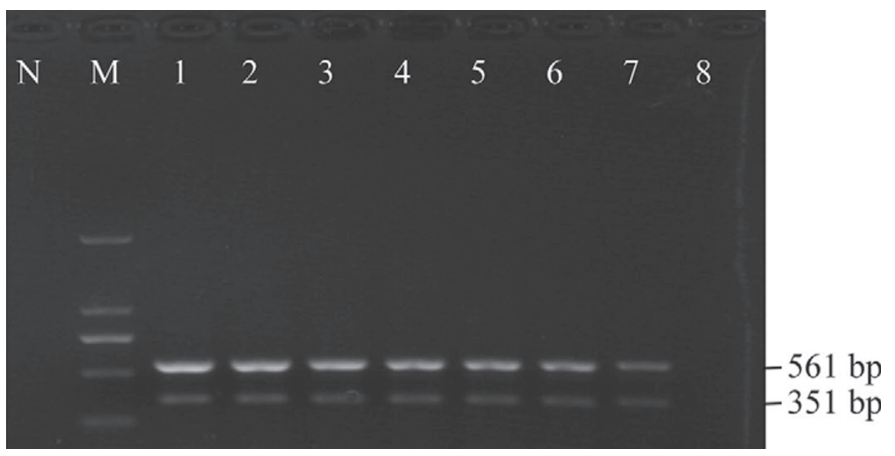

Figure 4. Detection of duplex PCR for ail and virF genes combined with nonselective enrichment. $\mathrm{M}=$ marker; $\mathrm{N}=$ negative control; lanes 1 to $8=$ enrichment for $18,16,14,12,10,8,6$, and $4 \mathrm{~h}$ at $28^{\circ} \mathrm{C}$, respectively.

net et al., 2001; Hanifian and Khani, 2012), but the relationship between these phenotypes and virulence is not completely consistent (Thoerner et al., 2003). The method described here could also distinguish pathogenic $Y$. enterocolitica strains with or without the virulence plasmid harboring the virF gene. Furthermore, instability of the virulence plasmid has been reported previously (Thoerner et al., 2003; Myers et al., 2006) and the plasmid can be easily lost under conditions of alkaline $\mathrm{pH}$ (e.g., pH 9.0; data not shown). Consequently, plasmid-borne virulence genes are not appropriate as the sole marker for specifically detecting pathogenic $Y$. enterocolitica strains in food samples.

\section{CONCLUSIONS}

In this study, we developed a duplex PCR targeting ail and virF virulence genes that could specifically and sensitively detect pathogenic $Y$. enterocolitica in frozen or refrigerated dairy products. A dot hybridization assay further confirmed the specificity of the duplex PCR. When combined with an enrichment step, the duplex PCR had high sensitivity. Detection of pathogenic $Y$. enterocolitica in commercial frozen or refrigerated dairy samples indicated that the duplex PCR was reli-

Table 1. Detection of Yersinia enterocolitica and pathogenic Y. enterocolitica in commercial refrigerated or frozen dairy samples by duplex PCR and China National Standard (2008; GB/T4789.8-2008)

\begin{tabular}{lcccc}
\hline & \multicolumn{3}{c}{ Detection by duplex $\mathrm{PCR}^{1}$} & \\
\cline { 2 - 4 } Dairy sample & ail & virF & ail and virF & $\begin{array}{c}\text { GB/T4789.8-2008 } \\
\text { method }\end{array}$ \\
\hline Refrigerated raw milk (14) & 1 & 0 & 0 & 2 \\
Ice cream (20) & 1 & 1 & 1 & 3 \\
Milk popsicles (18) & 2 & 0 & 0 & 3 \\
Refrigerated yogurt (14) & 0 & 0 & 0 & 2 \\
Frozen cheese (22) & 1 & 0 & 0 & 3 \\
Total (88) & 5 & 1 & 1 & 13 \\
\hline
\end{tabular}

${ }^{1}$ ail $=$ chromosome-borne attachment invasion locus gene; virF = plasmid-borne virulence factor $\mathrm{F}$ gene. 
Table 2. Virulence factors and phenotyping of pathogenic Yersinia enterocolitica isolates

\begin{tabular}{|c|c|c|c|c|}
\hline $\begin{array}{l}\text { Y. enterocolitica } \\
\text { strain }^{1}\end{array}$ & $\begin{array}{l}\text { Virulence } \\
\text { factor }^{2}\end{array}$ & Biotype & Serotype & Origin (food and region) \\
\hline Ye1,Ye3 & ail & $1 \mathrm{~B}$ & $0: 3$ & Meat (Xianan), chicken (Hefei) \\
\hline Ye4,Ye5, Ye6 & ail & 2 & O:5 & $\begin{array}{l}\text { Frozen dumpling (Haerbing), meat (Kunming), } \\
\text { meat gruel (Shanghai) }\end{array}$ \\
\hline Ye2 & ail & 3 & $\mathrm{ND}^{3}$ & Frozen chicken (Jinan) \\
\hline Ye7, Ye10, Ye12 & ail/virF & 2 & $\mathrm{O}: 8$ & $\begin{array}{l}\text { Frozen meat (Xianan), pork (Shantou), } \\
\text { frozen chicken (Lanzhou) }\end{array}$ \\
\hline $\mathrm{N}^{\boldsymbol{\Delta}}-\mathrm{Ye} 3$ & ail & 3 & O:9 & Milk popsicles (Hefei) \\
\hline $\mathrm{N}^{\boldsymbol{\Lambda}}-\mathrm{Ye} 4$ & ail/virF & 2 & $0: 3$ & Ice cream (Hefei) \\
\hline $\mathrm{N}^{\boldsymbol{\Lambda}}-\mathrm{Ye} 5$ & ail & 2 & O:5 & Frozen cheese (Hefei) \\
\hline
\end{tabular}

${ }^{1} \mathrm{~N}^{\mathbf{\Lambda}}=$ newly isolated from frozen and refrigerated dairy samples.

${ }^{2}$ ail $=$ chromosome-borne attachment invasion locus gene; virF $=$ plasmid-borne virulence factor $\mathrm{F}$ gene.

${ }^{3} \mathrm{ND}=$ nondetectable for $\mathrm{O}: 3, \mathrm{O}: 5, \mathrm{O}: 8, \mathrm{O}: 9$, and $\mathrm{O}: 1,2$.

able for detection of pathogenic $Y$. enterocolitica and for routine inspection and monitoring in refrigerated or frozen dairy samples.

\section{ACKNOWLEDGMENTS}

We gratefully acknowledge the financial support of the National Natural Science Foundation of China (Beijing, China; 31201292) and Guangdong Province, Chinese Academy of Science comprehensive strategic cooperation project (Guangzhou, China; 2012B090400017).

\section{REFERENCES}

Bhaduri, S., and B. Cottrell. 1997. Direct detection and isolation of plasmid-bearing virulent serotypes of Yersinia enterocolitica from various foods. Appl. Environ. Microbiol. 63:4952-4955.

Bottone, E. J. 1999. Y. enterocolitica: Overview and epidemiologic correlation. Microbes Infect. 1:323-333.

China National Standard. 2008. GB/T.4789.8-2008: Microbiological examination of Food hygiene-Examination of Yersina enterocolitica. Chinese National Standardization Management Committee, Beijing, China. (in Chinese)

EFSA-ECDC (European Food Safety Authority and European Center for Disease Prevention and Control). 2012. The European Union summary report on trends and sources of zoonoses, zoonotic agents and food borne outbreaks in 2010. EFSA J. 10:1-442.

Fredriksson-Ahomaa, M., and H. Korkeala. 2003. Low occurrence of pathogenic Yersinia enterocolitica in clinical, food, and environmental samples: A methodological problem. Clin. Microbiol. Rev. $16: 220-229$.

Grant, T., V. Bennett-Wood, and R. M. Robins-Browne. 1998. Identification of virulence-associated characteristics in clinical isolates of Yersinia enterocolitica lacking classical virulence markers. Infect. Immun. 66:1113-1120.

Guo, B. C., J. Zhan, Q. Hao, L. Q. Yan, X. Liu, M. Y. Xie, H. Q. Jing, X. Wang, and J. R. Liang. 2012. Analysis of the variation and changes of Yersinia enterocolitica in Ningxia area from 1984 to 2011. Zhonghua Yu Fang Yi Xue Za Zhi 46:879-882. (in Chinese).

Gürtler, M., T. Alter, S. Kasimir, M. Linnebur, and K. Fehlhaber. 2005. Prevalence of pathogenic Yersinia enterocolitica in fattening pigs. J. Food Prot. 68:850-854.
Hanifian, S., and S. Khani. 2012. Prevalence of virulent Yersinia enterocolitica in bulk raw milk and retail cheese in northern-west of Iran. Int. J. Food Microbiol. 155:89-92.

Harnett, N., Y. P. Lin, and C. Krishnan. 1996. Detection of pathogenic $Y$. enterocolitica using the multiplex polymerase reaction. Epidemiol. Infect. 117:59-67.

Huang, Y., X. Wang, Z. Cui, Y. Yang, Y. Xiao, L. Tang, B. Kan, J. $\mathrm{Xu}$, and H. Jing. 2010. Possible use of ail and foxA polymorphisms for detecting pathogenic Yersinia enterocolitica. BMC Microbiol. 10:211.

Jayarao, B. M., S. C. Donaldson, B. A. Straley, A. A. Sawant, N. V Hegde, and J. L. Brown. 2006. A survey of foodborne pathogens in bulk tank milk and raw milk consumption among farm families in Pennsylvania. J. Dairy Sci. 89:2451-2458.

Jegathesan, M., T. Paramasivam, K. Rajagopalan, and L. Loo. 1984 Yersina enterocolitica infection: First case report from Malaysia. Trop. Geogr. Med. 36:207-210.

Kandolo, K., and G. Wauters. 1985. Pyrazinamidase activity in Yersinia enterocolitica and related organisms. J. Clin. Microbiol. 21:980-982.

Kuhnert, P., J. Hacker, I. Muhldorfer, A. P. Burnens, J. Nicolet, and J. Frey. 1997. Detection system for Escherichia coli-specific virulence genes: absence of virulence determinants in B and C strains. Appl. Environ. Microbiol. 63:703-709.

Lantz, P. G., R. Knutsson, Y. Blixt, W. A. Al Soud, E. Borch, and P. Radstrom. 1998. Detection of pathogenic Yersinia enterocolitica in enrichment media and pork by a multiplex PCR: A study of sample preparation and PCR-inhibitory components. Int. J. Food Microbiol. 45:93-105.

Miller, V. L., J. B. Bliska, and S. Falkow. 1989. The ail locus is found uniquely in Yersinia enterocolitica serotypes commonly associated with disease. Infect. Immun. 57:121-131.

Miller, V. L., and S. Falkow. 1988. Evidence for two genetic loci in Yersinia enterocolitica that can promote invasion of epithelial cells. Infect. Immun. 56:1242-1248.

Myers, K. M., J. Gaba, and S. F. Al-Khaldi. 2006. Molecular identification of Yersinia enterocolitica isolated from pasteurized whole milk using DNA microarray chip hybridization. Mol. Cell. Probes $20: 71-80$.

Nilsson, A., S. T. Lambertz, P. Stalhandske, P. Norberg, and M. L. Daniellsson-Tham. 1998. Detection of Yersinia enterocolitica in food by PCR amplification. Lett. Appl. Microbiol. 26:140-144.

Prpic, J. K., R. M. Robins-Browne, and R. B. Davey. 1983. Differentiation between virulent and avirulent Yersinia enterocolitica isolates by using Congo red agar. J. Clin. Microbiol. 18:486-490.

Schiemann, D. A. 1987. Yersinia enterocolitica in milk and dairy products. J. Dairy Sci. 70:383-391. 
Skurnik, M., and H. Wolf-Watz. 1989. Analysis of the yopA gene encoding the Yop1 virulence determinants of Yersinia spp. Mol. Microbiol. 3:517-529.

Tan, L. K., P. T. Ooi, and K. L. Thong. 2014. Prevalence of Yersinia enterocolitica from food and pigs in selected states of Malaysia. Food Contr. 35:94-100.

Thoerner, P., C. I. Bin Kingombe, K. Bogli-Stuber, B. Bissig-Choisat, T. M. Wassenaar, J. Frey, and T. Jemmi. 2003. PCR detection of virulence genes in Yersinia enterocolitica and Yersinia pseudotu- berculosis and investigation of virulence gene distribution. Appl Environ. Microbiol. 69:1810-1816.

Wannet, W. J. B., M. Reessink, H. A. Brunings, and H. M. E. Maas. 2001. Detection of Pathogenic Yersina enterocolitica by a rapid and sensitive Duplex PCR assay. J. Clin. Microbiol. 39:4483-4486.

Yucel, N., and H. Ulusoy. 2006. A Turkey survey of hygiene indicator bacteria and Yersinia enterocolitica in raw milk and cheese samples. Food Contr. 17:383-388. 